Claremont Colleges

Scholarship@ Claremont

All HMC Faculty Publications and Research

HMC Faculty Scholarship

$1-1-2006$

\title{
Some Promising Approaches to Tumor-Immune Modeling
}

Lisette G. de Pillis

Harvey Mudd College

Ami E. Radunskaya

Pomona College

\section{Recommended Citation}

de Pillis, L. G. and A. E. Radunskaya. "Some Promising Approaches to Tumor-Immune Modeling." Mathematical Studies on Human Disease Dynamics: Emerging Paradigms and Challenges, AMS Contemporary Mathematics Series, Vol. 410, 2006, pp. 89-112.

This Article is brought to you for free and open access by the HMC Faculty Scholarship at Scholarship @ Claremont. It has been accepted for inclusion in All HMC Faculty Publications and Research by an authorized administrator of Scholarship @ Claremont. For more information, please contact scholarship@cuc.claremont.edu. 


\title{
Some Promising Approaches to Tumor-Immune Modeling
}

\author{
L.G. de Pillis and A.E. Radunskaya
}

\begin{abstract}
Mathematical models of tumor-immune interactions provide an analytical framework in which to address specific questions regarding tumorimmune dynamics. We present a brief summary of several approaches we are currently exploring to model tumor growth, tumor-immune interactions, and treatments. Results to date have shown that simulations of tumor growth using different levels of immune stimulating ligands, effector cells, and tumor challenge, are able to reproduce data from published studies. We additionally present some of our current efforts in the investigation of optimal control to aid in determining improved treatment strategies.
\end{abstract}

\section{Introduction}

Mathematical models of cancer progression and treatment have been under development by multiple research groups for a number of years, each new model tending, of necessity, to focus on simulating one or two important elements of the multifaceted process of tumor growth and response to therapy. In a cooperative effort with clinicians and research oncologists, we, too, have been investigating mathematical models of tumor growth in the context of immune system responses, with the goal of better understanding how the various aspects of growth, treatment, and the immune response interact with one another. We have developed a series of mathematical models, each evolving to address specific issues, all involving some representation of the immune system.

The question of whether the immune system plays a significant role in tumor development had been in the past a matter of debate. However, there is now a growing body of evidence showing that for certain forms of cancer, not only is the immune response relevant, but has been found to be essential if one is to observe tumor dormancy and cycling, and is also central to certain treatment programs $[21],[55],[50],[51],[65],[9]$.

1.1. Treatment Approaches. Chemotherapy, the administration of one or more drugs designed to kill tumor cells, is one of the most well established treatments for cancer. Many chemotherapy drugs target cells at the division stage. Unfortunately, this means that normal cells with a rapid cycle, such as those of the

Key words and phrases. Tumor modeling, Immune system modeling.

The first author was supported in part by NSF Grant DMS-0414011. 
hair, stomach lining, and those in the bone marrow where immune cells are produced, are also damaged by chemotherapy $[\mathbf{3 4}]$. One response to this is to combine several specific drugs that act on cells in different phases of their cycles. This is sometimes called combination therapy. Such combination therapy can also be beneficial when targeting tumor populations that may have immunity to one particular drug type, and to ensure that no one drug needs to be applied at levels toxic to the body [59].

Combination therapy can also include various forms of immunotherapy. Immunotherapy refers to a broad class of treatment techniques designed to boost the patient's immune system during treatment so the body's own defenses can be enlisted to help eradicate the cancer.

There are multiple approaches to boosting the immune system, among them II -2 growth factor injections (in order to increase the production of immune cells), the direct injection of highly activated specific immune cells, such as CD8 ${ }^{+} \mathrm{T}$ cells, into the blood stream, and vaccine therapy. The first two approaches focus on strengthening the immune system directly, whereas vaccine therapy challenges the body with a modified form of the cancer, stimulating the immune system to become activated against that specific invader.

1.2. Outline of Paper. Our investigations led us to develop our own series of mathematical models of cancer growth, which incorporate several key elements of the growth processes, immune responses, the effects of their mutual interactions, and components that allow us to simulate chemotherapy, general immunotherapy, and vaccine therapy. In certain cases, we have employed numerical optimal control methods to search for treatment protocols that are theoretical improvements to the standard protocols in use today.

The outline of this paper is as follows. In section 2, we present a three dimensional system of ODEs that describe the interactions among tumor cells, immuneeffector cells and normal cells. This model is then used to compare simulations of traditional chemotherapy strategies to those suggested by optimal control theory. In section 3, the ODE system is modified to explore the effect of tumor-specific and nonspecific cytotoxic immune cells. Mathematically the two cell types can be distinguished by their functional response form and model simulations are validated against published laboratory and clinical data. In section 4, we combine and extend these first two ODE models to include circulating lymphocytes, immunotherapy and chemotherapy. This larger model is used to simulate various combination therapy strategies. In section 5, spatial heterogeneity is added to the model through the implementation of a hybrid cellular-automata PDE approach, which can then be used to explore the effect of spatial cell and blood vessel distribution on the infiltration of immune cells, delivery of nutrients and therapy; and on tumor morphology. In section 6 , we describe another hybrid cellular-automata model. The goal of this model is to understand the implications of tumor induced acidity and hypoxia in the spread and treatment of the disease.

\section{Tumor, Normal, Immune Model with Optimal Control}

The first model we present incorporates three cell types: tumor cells $T(t)$, normal cells $N(t)$, and effector-immune cells $I(t)$. It also includes one drug treatment: the concentration of a cytotoxic chemotherapy $u(t)$. Model features include: 
rapy. Imto boost ses can be

long them une cells), $8^{+} \mathrm{T}$ cells, ; focus on lenges the to become

own series elements beractions, notherapy, al control ents to the

ree dimen, immuneulations of rol theory. or-specific pes can be e validated ind extend rerapy and on therapy rrough the in then be he infiltracorphology. roal of this hypoxia in

rol

; $T(t)$, nortreatment: lude:
(1) Immune response: immune cells, whose growth may be stimulated by the presence of the tumor, can destroy tumor cells through a kinetic process.

(2) Competition terms: normal cells and tumor cells compete for available resources.

(3) Optimal control theory for chemotherapy: a set of optimal therapy candidates is calculated to minimize the tumor population by the end of the fixed treatment period.

Full justification for each component can be found in $[11]$, with highlights provided here. The full system of equations is given by

$$
\begin{aligned}
\dot{I} & =s+\frac{\rho I T}{\alpha+T}-c_{1} I T-d_{1} I-a_{1}\left(1-e^{-u}\right) I \\
\dot{T} & =r_{1} T\left(1-b_{1} T\right)-c_{2} I T-c_{3} T N-a_{2}\left(1-e^{-u}\right) T \\
\dot{N} & =r_{2} N\left(1-b_{2} N\right)-c_{4} T N-a_{3}\left(1-e^{-u}\right) N \\
\dot{u} & =v(t)-d_{2} u
\end{aligned}
$$

The normal cells compete with the tumor cells for nutrients, and also serve as an indicator of the level of health, an important component when developing chemotherapy treatment strategies. Specifically, in this model normal cell levels are constrained to stay above a minimum threshold to eliminate those therapy protocols that would be too toxic. We assume an initially small tumor mass, that is, a tumor size that is close to zero relative to carrying capacity. Consequently, the choice of the mathematical growth law to represent tumor cell growth dynamics does not significantly affect the qualitative behavior of the model. We compared the results of the simulations of our system using a variety of standard growth laws, and all gave results that were qualitatively similar. The logistic and Von Bertalanffy growth laws provided the best fit to a specific data set, but logistic growth required fewer parameters than Von Bertalanffy, see Figure (1). 'Therefore, we use logistic growth in this model.

In the absence of a tumor, the immune cells are produced at a constant rate $s$, and have a natural per capita death rate $d_{1}$, resulting in a long-term population size of $s / d_{1}$ cells. The presence of tumor cells stimulates the immune response, represented by the positive nonlinear growth term for the immune cells $\frac{\rho I(t) T(t)}{\alpha+T(t)}$, where $\rho$ and $\alpha$ are positive constants. This type of response term is of the same form as the terms used in the respective models of Kuznetsov et al. (1994), Kirschner and Panetta (1998), and Owen and Sherratt (1998). As a function of $T$, the response term is positive, increasing, and concave. Furthermore, the reaction of immune cells and tumor cells can result in either the death of tumor cells or the inactivation of the immune cells, resulting in the two competition terms. Both tumor cells and the normal cells obey a logistic growth law, with parameters $r_{i}$ and $b_{i}$ representing the per capita growth rates and reciprocal carrying capacities of the tumor and normal cells, $i=1,2$ respectively. In addition, there are two terms representing the competition for resources between tumor and host cells.

To add the effect of drug on the system, we denote by $u(t)$ the concentration of drug at the tumor site at time $t$. We assume that the drug kills all types of cells, but that the kill rate differs for each type of cell. Letting $F(u)$ be the fractional cell kill for a given amount of drug, $u$, at the tumor site, we use $F(u)=a_{i}\left(1-e^{-u}\right)$, where $a_{i}, i=1,2,3$ are the three different response coefficients. This form reflects 

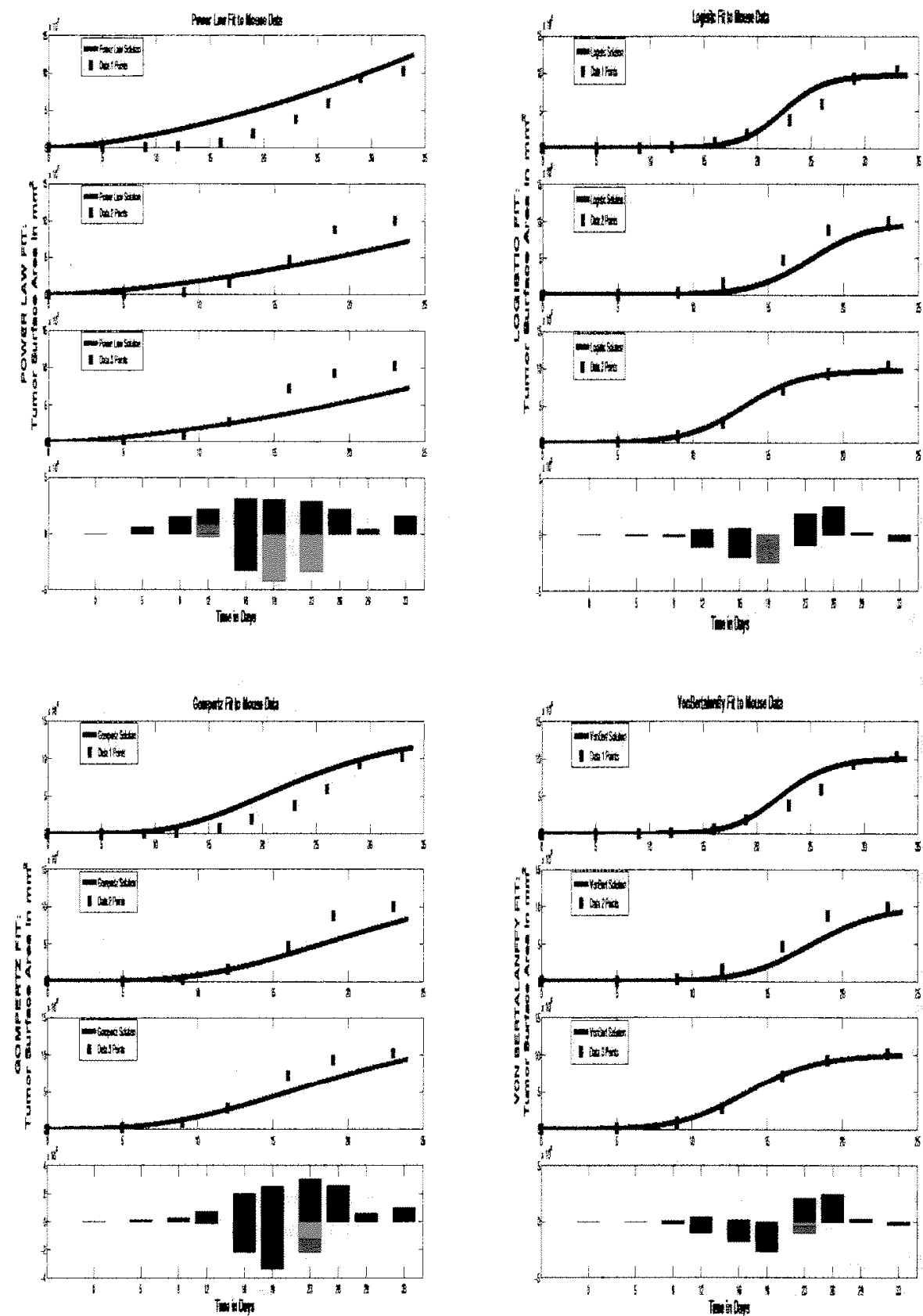

Objec

where $K_{1}$ and $K$ reduces to $J(v)=$

In this case, $t$ l control leads to sc on one of two valu dose allowed. Det. found in [11]. The method implemen also be found usir Problems with no: solved with tradit: or the collocation for solving optime such as the develo] or humoral immu

\subsection{Compa}

Figure 1. A comparison of various growth laws to three tumor growth data sets from published data in $[\mathbf{1 5}]$. Each data set represents tumor volume growth over time, with three different inital tumor burdens: $10^{4}, 10^{5}$, and $10^{6}$ cells. The bottom graph in each set shows the superimposed residuals (model prediction minus data point) for all three data sets. The smallest pointwise residuals are achieved with the Von Bertalanffy and the Logistic growth models, hence these provide the best fit.

the protocol sugg be administered $\mathrm{c}$ days. Standard pr several hours, wit?

In $[\mathbf{1 1}]$ and [ optimal control ch 
the dose-response curves suggested in [27]. The rate of change of $u(t)$ is determined by the dose given, $v(t)$, and a per unit elimination rate of the drug.

A full stability analysis is carried out on this model in [11] and [12].

2.1. Formulation of the Optimal Control Problem. In this model, we have the ability both to simulate a predetermined course of treatment, and to employ the theory of optimal control to help automate the process of determining improved treatment strategies. Optimality in treatment might be defined in a variety of ways. Some studies, for example, have chosen to minimize the total amount of drug administered while either maximizing healthy cells or minimizing cancer cells $[69],[68],[70]$. In contrast, in this model the optimal control problem with state constraints is framed as follows: for a fixed time interval $\left[0, t_{f}\right]$, find the points within that interval at which the drug should be administered so that the number of tumor cells is minimized, while the number of healthy cells is kept above a prescribed threshold. In particular, we must determine the function $v(t)$ that will minimize the number of tumor cells up to some specified time, $t_{f}$, with the constraint that normal cells are not overly depleted. The units of cells are normalized, so the carrying capacity of normal cells is 1 (i.e., $b_{2}=1$ ). We require that the number of normal cells stay above three-fourths of the carrying capacity, or $N(t) \geq .75$ for all $t$. Therefore, in the language of optimal control theory we
have:

$$
\begin{aligned}
\text { Objective Functional: } & J(v)=K_{1} T\left(v, t_{f}\right)+K_{2} \frac{1}{t_{f}} \int_{0}^{t_{f}} T(v, t) d t \\
\text { Constraint: } & N(t) \geq .75 \quad 0 \leq t \leq t_{f}
\end{aligned}
$$

where $K_{1}$ and $K_{2}$ are prioritizing weights. Note that this objective functional reduces to $J(v)=T\left(v, t_{f}\right)$ if $K_{1}=1$ and $K_{2}=0$

In this case, the objective functional is linear in the control. The linearity of the control leads to solutions that are "bang-bang". That is, the drug dose $v(t)$ takes on one of two values: $v$ is either zero, or takes on the value $v_{\max }$, the maximum drug dose allowed. Details of the derivation of the characterization of this solution can be found in [11]. The numerical solution of this problem was solved using a collocation method implemented in the software DIRCOL v1.2 [75]. Bang-bang solutions can also be found using alternate approaches, such as genetic algorithms $[\mathbf{7 4 , 4 3 , 4 6 ]}$. Problems with nonlinear controls and smooth solutions may be more appropriately solved with traditional methods such as two-point-boundary value problem solvers, or the collocation technique of DIRCOL. A variety of approaches have been taken for solving optimal control problems focusing on other areas of cancer treatment, or or humoral immunotherapy. See, for example, $[42,71,19,25]$.

2.2. Comparison with Standard Protocols. In most of our experiments, the protocol suggested by the optimal control algorithm dictated that the drug be administered continuously over relatively long periods of time-on the order of days. Standard protocol is to administer the drug for a short time, on the order of several hours, with periodically repeated treatments every few weeks.

In [11] and [12] a series of experiments comparing pulsed chemotherapy to optimal control chemotherapy are presented, while [12] also discusses the basins of 

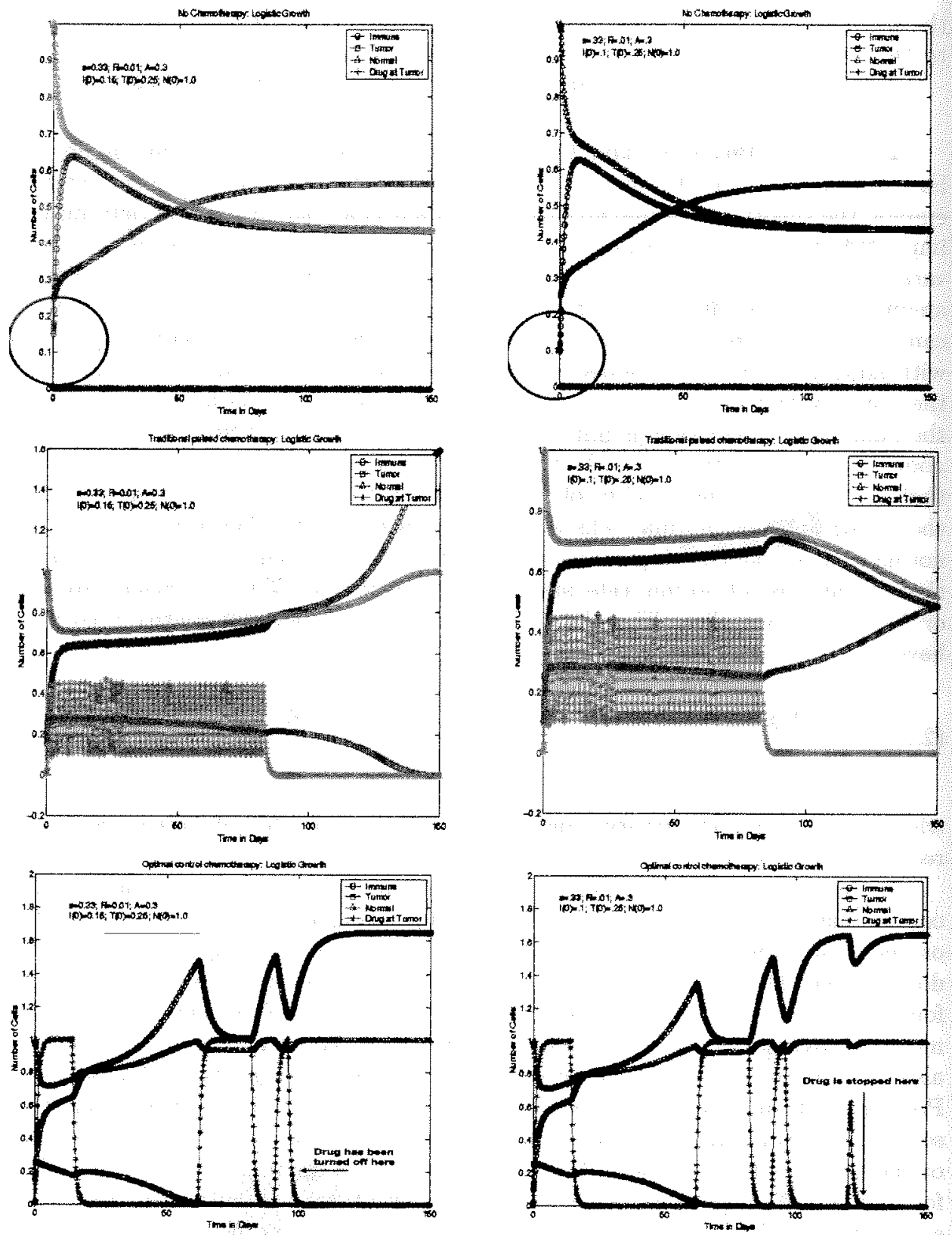

$\mathrm{I}(0)=0.15$

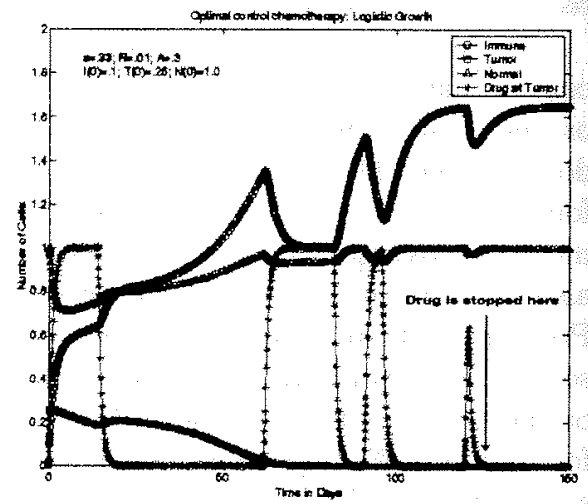

$\mathrm{I}(0)=0.1$

Figure 2. Comparison of tumor growth with two initial immune strengths. Left: $I(0)=0.15$. Right: $I(0)=0.10$. Top Row: No chemotherapy: in both cases, the tumor cell population grows; Middle Row: Traditional pulsed treatment: with $I(0)=.15$, the tumor is controlled, but with $I(0)=.10$, the tumor grows; Bottom Row: Optimal control treatment: in both cases the tumor is controlled and does not regrow, even after treatment is stopped.

attraction to a her iments highlightin system determines is, drive the cancer time with no drug 2, Middle Row, we the tumor when t tem is only slightl the optimal contr 2, Bottom Row. population into th See [12] for an ani

2.3. Possible this work is to inve final time, and th basin of attraction cell cycle to allow $f$ equations can be $\epsilon$ made in this direc [11] and [12].

\section{Tumor, Nons}

This next mod ponents of immun differing roles of th cells and the speci mor cells. NK cells and destroy tumo killer cells are tho cer by killing abn critical subpopulat vided previous sen cell populations by

- $T(t)$, tur

- $N(t)$, tot.

- $L(t)$, tota

This model is 1 et al. [15], in whi of immune stimule human data provi melanoma are trea and the human st growth rates and $\epsilon$ dynamics of tumor development of prc 
attraction to a healthy zero tumor equilibrium. Figure 2 presents numerical experiments highlighting a hypothetical case in which the initial strength of the immune system determines whether traditional chemotherapy can "cure the patient", that is, drive the cancer population to zero. Figure 2, Top Row, shows tumor growth over time with no drug intervention for two levels of initial immune strength. In Figure 2 , Middle Row, we see that traditional pulsed chemotherapy successfully eliminates the tumor when the immune system is stronger, but fails when the immune system is only slightly weaker. In the case for which traditional chemotherapy fails, the optimal control chemotherapy strategy is successful, as highlighted by Figure 2, Bottom Row. That is, the optimal control strategy is able to push the tumor population into the zero basin of attraction even with a weakened immune system. See [12] for an analysis of the basins of attraction of this system.

2.3. Possible Extensions of the T-N-I Model. One natural extension of this work is to investigate alternate objective functionals, including those with free final time, and those whose focus is simply to drive the system into a desirable basin of attraction. Another extension to this model would take into account the cell cycle to allow for the modeling of cycle specific chemotherapy. Delay differential equations can be employed to model cell-cycle specificity. Some progress has been made in this direction in [60]. Further ideas for model extensions can be found in $[\mathbf{1 1}]$ and $[\mathbf{1 2}]$.

\section{Tumor, Nonspecific-Effector, Specific-Effector Response Model: the $T-N-L$ model}

This next model focuses more specifically on the interaction of particular components of immune system with the growing cancer. This model highlights the differing roles of the nonspecific-effector cells, represented by Natural Killer (NK), cells and the specific-effector cells, represented by $\mathrm{CD} 8^{+} \mathrm{T}$ cells, in suppressing tumor cells. NK cells are a constituent of innate immunity, and are able to recognize and destroy tumor cells, among others, independent of prior exposure. Natural killer cells are thought to play a key role in preventing the development of cancer by killing abnormal cells before they multiply and grow. $\mathrm{CD}^{+} \mathrm{T}$ cells are a critical subpopulation of T-lymphocytes that can be cytotoxic to tumor cells provided previous sensitization has occurred. In the equations, we denote the three cell populations by:

- $T(t)$, tumor cell population at time $t$

- $N(t)$, total level of natural killer cell effectiveness at time $t$

- $L(t)$, total level of tumor specific $\mathrm{CD} 8^{+} \mathrm{T}$ cell effectiveness at time $t$

This model is based upon and validated by experimental studies by Diefenbach et al. [15], in which mouse tumor cell lines are modified to express higher levels of immune stimulating NKG2D ligands. The model is further validated against human data provided in Dudley et al. [17], in which subjects with metastatic melanoma are treated with highly selected tumor-reactive $\mathrm{T}$ cells. Both the mouse and the human studies provide vital experimental information regarding tumor growth rates and effector to target lysis rates. The model is used to explore the dynamics of tumor rejection, the specific role of the NK and CD8+T cells, and the development of protective immunity to subsequent tumor rechallenge. 
3.1. Model Development. The specific biological assumptions we took into account when developing the model equations are based on both accepted knowledge of immune system function and conclusions stated in [15] and [17]. The assumptions include:

(1) The tumor cells grow logistically in the absence of an immune response. See section 2.

(2) Both natural killer cells (NK) and $\mathrm{CD}^{+} \mathrm{T}$ cells can kill turnor cells.

(3) Tumor cells have the potential to engender cytolytic activity in previously naive and non-cytotoxic cells.

(4) As part of innate immunity, NK cells are always present and active in the system, even in the absence of tumor cells.

(5) As part of specific immunity, tumor-specific $\mathrm{CD} 8^{+} \mathrm{T}$ cells are produced and recruited once tumor cells are present.

(6) Each natural killer cell and CD8 ${ }^{+} \mathrm{T}$ cell will eventually become inactivated after some number of encounters with tumor cells.

Detailed justification of each assumption can be found in [14].

3.2. Model Equations. Using the list of assumptions from above, we describe the system as three coupled differential equations, where each equation gives the rate of change of the particular cell population in terms of growth and death, cell-cell kill, cell recruitment, and cell inactivation.

The mathematical forms of the growth and death terms for tumor and immune cell populations reflect assumptions (1), (4) and (5). Assumption (2) is reflected in the cell-cell kill term, assumption (3) gives rise to the effector cell recruitment terms, and assumption (6) is incorporated through the effector inactivation terms.

Immune recruitment terms are generally assumed to be of a Michaelis-Menten form, (see, e.g., $[\mathbf{4 0}]$ in which Michaelis-Menten dynamics are derived for immune cell recruitment by cancer cells). These dynamics are commonly used in mathematical tumor models that include an immune component, since they allow for a saturation effect (see, e.g., $[\mathbf{3 7}]$ ). In the case of the CD8 ${ }^{+} \mathrm{T}$ cells, in addition to being recruited by interactions with T-cell processed tumor cells through a MichaelisMenten dynamic, additional CD8 ${ }^{+} \mathrm{T}$ cells are stimulated by the interaction of NK cells with tumor cells. This NK stimulation is represented by the $r N T$ term in equation (3.3). The term $r N T$, representing a fraction of the number of interactions between NK cells and tumor cells, is the vehicle through which we model the fact that the specific immune response of the $\mathrm{CD} 8^{+} \mathrm{T}$ cells is activated only after the activation of the earlier response of innate immunity. To determine the fractional cell kill dynamics, data from standard four hour chromium release assays published in [15] and $[\mathbf{1 7}]$ were used.

The fractional cell kill term for the NK cells was assumed to be proportional to the size of the NK cell population. This assumption was consistent with all the data we examined. However, the same assumption was not consistent with the data for tumor-specific $\mathrm{CD}^{+} \mathrm{T}$ cells. We therefore introduced a new functional form for the $\left(\mathrm{CD} 8^{+} \mathrm{T}\right)$-tumor kill term, represented by $D$ in equation (3.1), given explicitly in equation (3.4) below. This term we say is of a "rational" form. Since this term is an innovation in this model, its derivation is specifically addressed in the next section.

Substituting specific mathematical forms for each of the growth, death, recruitment and inactivation terms yields the following system of equations: 
e took into ited knowl[17]. The

e response.

cells.

previously

stive in the

produced

inactivated

ve, we deation gives and death,

ad immune is reflected ecruitment ion terms. lis-Menten or immune in matheallow for a tion to beMichaelistion of NK $T$ term in of interacwe model vated only zrmine the zase assays

oportional rith all the $h$ the data al form for t explicitly : this term a the next h, recruit-

$$
\begin{aligned}
\frac{d T}{d t} & =a T(1-b T)-c N T-D \\
\frac{d N}{d t} & =\sigma-f N+\frac{g T^{2}}{h+T^{2}} N-p N T \\
\frac{d L}{d t} & =-m L+\frac{j D^{2}}{k+D^{2}} L-q L T+r N T
\end{aligned}
$$

where

$$
D=d \frac{\left(\frac{L}{T}\right)^{\lambda}}{s+\left(\frac{L}{T}\right)^{\lambda}} T .
$$

Reference [12] provides a detailed listing of the parameters in this model, along with their units, descriptions, numerical values for the simulations, and reference sources from which these values were taken. Detailed development of all terms, except for the new fractional cell kill term $D$ discussed below, can be found in [11].

3.2.1. Fractional Cell Kill. A crucial component of the model equations is the set of terms describing the interaction or "competition" between the tumor cells and either the $\mathrm{NK}$ cells or the $\mathrm{CD} 8^{+} \mathrm{T}$ cells. It is common to assume that that the action of the effector cells is to reduce the tumor cell population through a massaction dynamic (see for example, $[52,20,49,41,40,11]$ ). In our case, the effect of the NK cells on the tumor cell population would be expressed with the term $-c N T$, while the form of the CD ${ }^{+} \mathrm{T}$ cell effect would be $-d L T$, where $c$ and $d$ are proportionality parameters determined through experiment. As discussed below, we found that although this simple mass-action term was sufficient to reproduce in simulations the experimental interaction of NK cells with tumor cells, it did not accurately reflect the action of the $\mathrm{CD} 8^{+} \mathrm{T}$ cells.

We performed data fitting experiments using a generalization of the mass-action term: a power term that allows for exponential growth. Using cell lysis data from [15], we employed a least squares fit to find the parameters $c$ and $\nu$ in $c N^{\nu} T$, and $d$ and $\lambda$ in $d L^{\lambda} T$. Details can be found in [14]. We found that the best-fit exponent for the NK kill term was $\nu \approx 1$, so we chose to keep the product form, $-c N T$, to describe the effect of the NK cells on tumor cells. In fact, the optimal value of $c$ determined using our algorithm reproduced the lysis rate data extremely well (see [14]).

However, when fitting for parameters $d$ and $\lambda$ for the $\mathrm{CD} 8^{+} \mathrm{T}$ cell kill term, we found that the power form produced values for the lysis rate that were not particularly good fits to the data provided in [15]. Instead, we found that we could produce curves that better fit the data by allowing this term to have the rational form given in equation (3.4), for which we also had to determine parameter $s$. In (3.4), the exponent $\lambda$ represents how the lysis rate depends on the effector:target ratio, the parameter $s$ affects the steepness of the curve, and parameter $d$ gives the maximum lysis rate. The selection of the rational form of the cell-kill term is further supported by the fact that both in vitro and in vivo experiments indicate that percent lysis appears to be a function of the ratio of $\mathrm{CD} 8^{+} \mathrm{T}$ cells to tumor cells, explaining the appearance of $(L / T)$. Furthermore, the data indicate that the percent of cells lysed never exceeds a maximum, a saturation effect that is reflected by the rational form given in equation $(3.4)$. 
This saturation effect highlights the fact that the NK cells and CD ${ }^{+} \mathrm{T}$ cells are interacting with tumor cells in a qualitatively different way, since there is no saturation level for the NK cell competition term. It may be that the NK-cell kill rate could achieve saturation as well in theory, but in practice this does not occur. On the other hand, it may be that the antigen-specific T-cells follow this curve to saturation because they are targeting a specific tumor type, and are therefore more effective in terms of cell-cell interactions.

3.3. New Functional Forms. In contrast to NK cells, CD $8+\mathrm{T}$ cells have to be primed in order to be activated. When determining the $\mathrm{CD} 8^{+} \mathrm{T}$ kill term $D$, there were four different data sets to be fitted. These came from first priming with either control-transduced cells or with ligand-transduced cells, then challenging with either control-transduced or ligand-transduced cells. In the model, these cases are distinguished by the estimates of the parameters, $d$ and $\lambda$, which changed by a factor of up to five. Thus, the estimated values of these parameters as components of the rational cell kill term $D$ may be highly significant factors distinguishing between tumors that are weakly and strongly antigenic.

3.3.1. Functional and Numerical Response. In ecology literature, predator-prey dynamics have been modeled and studied in a variety of ways, frequently framed using a functional-numerical response approach, in which the rate of prey consumption by an average predator is known as the "functional response." The form of the functional response plays a significant role in determining dynamic stability, responses to environmental influences, and the nature of indirect effects of external resources on the predator-prey pair. The functional response is often classified as either prey-dependent, in which prey density alone determines the response, or predator-dependent, in which both the predator and prey populations are components in the response.

It is not unreasonable to consider the effector-immune to tumor interaction as a type of predator-prey interaction, in which case $D$ can be framed in the ecological functional-numerical response format. Earlier models of tumor-immune interactions, including that of section 2 and $[11]$, and the dynamic representing the $\mathrm{NK}$-cell to tumor interaction, have employed essentially prey-dependent type dymamics to describe the immune-tumor interaction. Similarly, until recently, predation theory in ecology was dominated by prey-dependent models [1]. More recently, however, ratio-dependent functional response models, a particular subtype of the predatordependent model, have been shown to be more appropriate in many ecological settings $[1,2]$. The ratio-dependent model assumes that the response depends on the ratio of the prey population size to the predator population size, and not on the absolute number of each species. A cell lysis term of the form $D$ is new in this tumor modeling context, and does not fit into the traditional prey-dependent categorization. However, since the dynamic we discovered through our data fitting process led us to a ratio-dependent term, it is interesting to observe that our problem can be recast in the form of a predator-ratio-dependent functional response. Namely, ignoring the action of the NK cells, and letting our functional response term be

$$
g(T, L)=d \frac{(L / T)^{\lambda-1}}{s+(L / T)^{\lambda}}
$$

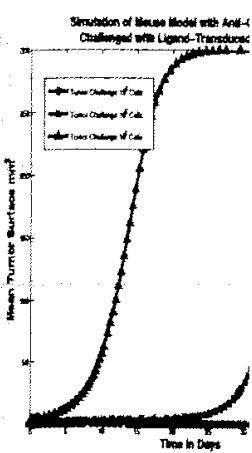

FIGURE 3. over time pleted; Ce ponents ar tween the mean tumc whereas in the maxim

and our numerical I for $T$ and $L$ can be

$$
\frac{d T}{d t}
$$

where $f_{1}$ is an int response of the pre

While it is not $\mathrm{i}$ biologically, the use is quite common, ar hoped that future $\mathrm{i}$ : mechanisms that gi

3.4. Model S ical model can be 1 reproduce with a co results of three sets For the experiment or $10^{6}$ ligand-trans For our in silico sim three levels of tum time in response to CD $8+\mathrm{T}$ cell activit CD ${ }^{+} \mathrm{T}$ cells. This immune response. of $10^{5}$ cells or more simulated tumor gr sence of $\mathrm{NK}$ cells, $r$ 
and $\mathrm{CD} 8^{+} \mathrm{T}$ cells $r$, since there is no at the NK-cell kill his does not occur. ollow this curve to are therefore more

$38^{+} \mathrm{T}$ cells have to $38^{+} \mathrm{T}$ kill term $D$, first priming with then challenging model, these cases hich changed by a ers as components ors distinguishing

ure, predator-prey frequently framed $\therefore$ of prey consumpise." The form of dynamic stability, it effects of exteris often classified $s$ the response, or ations are compo-

nor interaction as led in the ecologir-immune interacsnting the NK-cell type dynamics to predation theory ecently, however. 3 of the predator many ecological sonse depends on size, and not on orm $D$ is new in 1 prey-dependent h our data fitting ve that our prob. ctional response actional response
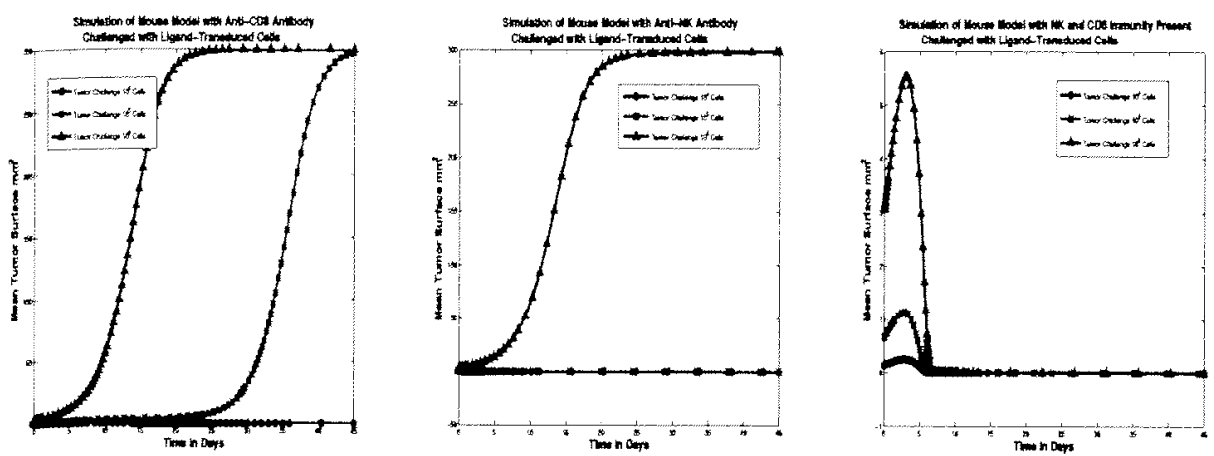

FIgURE 3. Simulations of (tumor-cell)-(immune-cell) interactions over time in three different situations. Left: $\mathrm{CD} 8^{+} \mathrm{T}$ cells are depleted; Center: NK cells are depleted; Right: all immune components are intact. Note the difference in the vertical scale between the left and center panels, and the right panel. Maximum mean tumor surface area in the left and center panels is $300 \mathrm{~mm}^{2}$, whereas in the right panel in which the tumor is not established, the maximum surface area is never greater than $6 \mathrm{~mm}^{2}$.

and our numerical response be a Michaelis-Menten type function of $g$, the equations for $T$ and $L$ can be written in a standard functional-numerical response form:

$$
\frac{d T}{d t}=T f_{1}(T)-L g(T, L) ; \quad \frac{d L}{d t}=L f_{2}(g(T, L))-m L
$$

where $f_{1}$ is an intrinsic growth function for $T$, and $f_{2}$ represents the numerical response of the predator $L$.

While it is not immediately clear what the individual components of $D$ represent biologically, the use of phenomenological dynamics in modeling biological processes is quite common, and can serve to provide predictive capabilities in the model. It is hoped that future investigations will elucidate the the underlying immune response mechanisms that give rise to the form of $D$.

3.4. Model Simulations. Simulations generated by a validated mathematical model can be used to detect thresholds for immune efficacy. In Figure 3, we reproduce with a computational solution of our mathematical model the qualitative results of three sets of experiments that were presented in Figure 2, page 167 of [15]. For the experiments in [15], groups of mice were challenged with either $10^{4}, 10^{5}$ or $10^{6}$ ligand-transduced tumor cells, and then tumor establishment was tracked. For our in silico simulations, we also challenge the mathematical system with these three levels of tumor cells. Figure 3 (left) shows simulated tumor cell growth over time in response to these three initial levels of tumor burden in the absence of $\mathrm{CD} 8+\mathrm{T}$ cell activity, reflecting the experiments in which the mice were depleted of CD $8^{+} \mathrm{T}$ cells. This simulation represents a system lacking a strong antigen-specific immune response. The system can control a small tumor, but tumor challenges of $10^{5}$ cells or more escape the immune system's control. Figure 3 (center) shows simulated tumor growth outcomes for the same three experiments done in the absence of NK cells, reflecting the experiments with mice depleted of NK cells. The 
system is now able to control initial tumor burdens of up to $10^{5}$ cells, but a larger challenge of $10^{6}$ cells escapes immunosurveillance. Figure 3 (right) shows simulated results with both $\mathrm{NK}$ and $\mathrm{CD} 8^{+} \mathrm{T}$ cells active, reflecting the experiments on mice with intact immune systems. With both the $\mathrm{NK}$ cells and the $\mathrm{CD} 8^{+} \mathrm{T}$ cells working together, initial tumor burdens of up to $10^{6}$ cells are controlled.

Our new tumor-immune interaction dynamic form provides a good fit with experimental data resulting from priming and rechallenge with different combinations of tumor cell types. This model suggests the value in continuing to research the mechanisms by which $\mathrm{NK}$ cells and $\mathrm{CD} 8^{+} \mathrm{T}$ cells induce tumor cell lysis. The functional descriptions of these cell populations suggest that further laboratory tests may aid in determining why the two types of immune cells give rise to such different cell interaction dynamics. We hypothesize that the more effective the immune cell kill is, the more closely it follows a rational law dynamic.

3.5. Possible extensions of the $T-N-L$ model. The model currently in cludes no self-regulatory terms in the equations, or down-regulation of an activated immune response. This issue is currently undergoing active attention. These experimental and simulated results, observed together with the cell lysis data presented here and the parameter sensitivity analysis presented in [14], highlight the importance of $\mathrm{CD}^{+} \mathrm{T}$ cell activation on final outcome. Model results appear to indicate that in order to promote tumor regression, it may be necessary (although perhaps not sufficient) to focus on increasing $\mathrm{CD} 8^{+} \mathrm{T}$ cell activity. In fact, we propose that there may be a direct positive correlation between the patient-specific efficacy of the $C D 8^{+} T$ cell response as measured by cytotoxicity assays, and the likelihood of a patient responding favorably to immunotherapy treatments. This hypothesis could be tested in clinical studies.

\section{Model with IL-2, Circulating Lymphocytes and Chemotherapy}

This model incorporates key elements of both of the previous two models: specific effector response, along with a way to implement optimal control. With this model we are able to explore combination chemo-immunotherapy, and we have a foundation for implementing optimal control (this last is currently under development). The model describes the kinetics of four populations (tumor cells and three types of immune cells), as well as two drug concentrations in the bloodstream, using a series of coupled ordinary differential equations. The populations, $T(t), N(t)$ and $L(T)$ are the same as in the previous model. Additionally we have:

- $C(t)$, number of circulating lymphocytes (or white blood cells)

- $M(t)$, chemotherapy drug concentration in the bloodstream

- $I(t)$, immunotherapy drug concentration in the bloodstream (e.g. IL -2$)$.

In contrast to the first model in which the generic normal cell population is used as a state constraint for the optimal control problem, here the circulating lymphocyte levels are incorporated to be used as a measure of patient health (see, e.g., $[53,48$, 30]). The advantage to moving to the use of circulating lymphocytes is that these can actually be measured in a patient's bloodstream, so realistic parameters can be obtained. The full system of six differential equations incorporates the same fractional cell kill dynamic, $D=d(L / T)^{\lambda} T /\left(s+(L / T)^{\lambda}\right)$ discussed in detail in 
out a larger $\mathrm{s}$ simulated nts on mice ells working

fit with exmbinations -esearch the 3. The funcratory tests uch different immune cell

vurrently inan activated These experta presented $t$ the impor$r$ to indicate ugh perhaps propose that ic efficacy of re likelihood is hypothesis

\section{therapy}

models: spe3l. With this id we have a Ider developolls and three stream, using ( $t), N(t)$ and

\section{lls)}

( e.g. IL-2),

ion is used as g lymphocyle , e.g. $[53,48$. ; is that these urameters can ttes the same $\mathrm{d}$ in detail in section 3. The system becomes:

$$
\begin{aligned}
\frac{d T}{d t}= & a T(1-b T)-c N T-D-K_{T}\left(1-e^{-M}\right) T \\
\frac{d N}{d t}= & e C-f N+g \frac{T^{2}}{h+T^{2}} N-p N T-K_{N}\left(1-e^{-M}\right) N \\
\frac{d L}{d t}= & -m L+j \frac{D^{2} T^{2}}{k+D^{2} T^{2}} L-q L T+\left(r_{1} N+r_{2} C\right) T \\
& -u N L^{2}-K_{L}\left(1-e^{-M}\right) L+\frac{p_{I} L I}{g_{I}+I}+v_{L}(t) \\
\frac{d C}{d t}= & \alpha-\beta C-K_{C}\left(1-e^{-M}\right) C \\
\frac{d M}{d t}= & -\gamma M+v_{M}(t), \quad \frac{d I}{d t}=-\mu_{I} I+v_{I}(t)
\end{aligned}
$$

This model allows for the administration of traditional chemotherapy as well as both cellular and humoral immunotherapy. Chemotherapy is administered through the term $v_{M}$ in the equation for the medicine $M$, and affects the other cell populations with a per cell kill rate saturating at the coefficient $K_{j}$, where $j=T, N, L, C$. A tumor infiltrating lymphocyte (TIL) drug intervention term, $v_{L}$, in the equation for the CD8 ${ }^{+} \mathrm{T}$ cells, $L$, is a function of time. When $v_{L}(t)$ is non-zero, this represents the administration of cellular immunotherapy in which the immune-effector cell levels are boosted directly by the addition of antigen-specific cytolytic T-cells. Similarly, the drug intervention term $v_{I}$ in the equation for $I$ reflects the amount of a humoral immune-stimulating drug, such as IL-2, given over time.

A full description of the equations, their derivation, and a stability analysis can be found in $[\mathbf{1 0}]$. In [10] a variety of computational experiments is also presented, including a numerical investigation of the theoretical potential for combining vaccine therapy with chemotherapy. In one case, a theoretical patient is given a detectable tumor of size $2 \times 10^{7}$ and a "healthy" immune system of $3 \times 10^{5} \mathrm{NK}$ cells, $10^{2}$ CD $8^{+} \mathrm{T}$ cells, and $10^{10}$ circulating lymphocytes. This is a case in which patient's immune system is not strong enough to handle a $10^{7}$ size tumor on its own. As shown in Figure 4 (left) the body cannot handle this size tumor even when treated with aggressive pulsed chemotherapy for 50 days. Additionally, vaccine therapy, represented by changes to the original parameter set after 10 days, fails to control the tumor. This simulation is pictured in Figure 4 (center). Only the combination of both treatments can kill a tumor of this magnitude, as shown in Figure 4 (right). The parameters for these simulations are all provided in [10].

Such dramatic tumor regression is still uncommon among most patients. The result is sensitive to the choice of tumor and patient parameters, as well as to the timing of the treatments, as described in [10]. It has been noted, however, in the clinical studies of Wheeler [76], that over a period of 50 days, combination therapy provoked a drastic $(>50 \%)$ tumor regression in three of thirteen glioma patients. Wheeler points out that these tumor regression outcomes are rare in the literature. This mathematical model is able to reflect these positive clinical responses to emerging combination therapies, and could be used to identify aspects of the immune response that are potentially critical to these positive outcomes. 

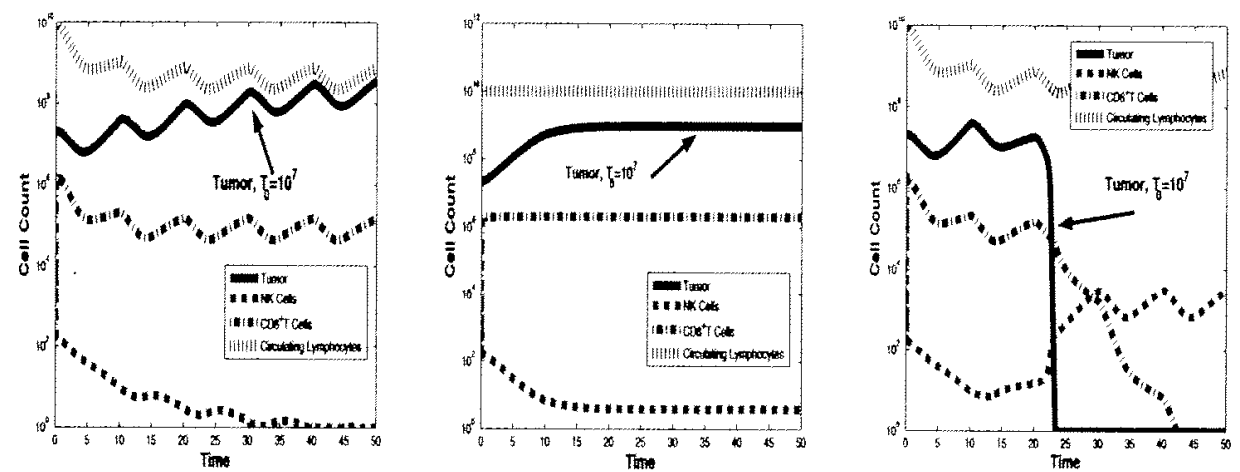

Figure 4. Human data. Left: Chemotherapy alone cannot kill a $2 \times 10^{7}$ tumor with an immune system of $3 \times 10^{5}$ NK cells, 100 $\mathrm{CD} 8^{+} \mathrm{T}$ cells, and $10^{10}$ circulating lymphocytes. Chemotherapy is administered for 3 consecutive days in a 10 day cycle. Center: Vaccine therapy alone cannot control the tumor with the same initial conditions as given in Left figure. Right: Combination therapy effectively controls the tumor.

\section{Hybrid PDE-CA Models}

For solid tumors, spatial heterogeneity becomes an important factor in both growth and treatment. Therefore, the next step in model development extends the system of ordinary differential equations to a system that includes a spatial component. This can be realized in more than one way. Traditionally, this has been accomplished through the implementation of partial differential equations (PDEs), where both space and time are independent variables.

Alternately, cellular automata (CA) modeling allows for the inclusion of spatial heterogeneity, employing a discrete dynamical system on a discrete, finite state space. The model presented in [45] allows both temporal and two-dimensional spatial evolution of the system and is comprised of biological cell metabolism rules derived from both the experimental and mathematical modeling literature. In our model, as in many CA models of tumor growth, some of the state variables are continuous and hence the modifier "hybrid". Here we consider the nutrient limited growth of an avascular tumor and the dynamic interplay of both the specific (innate) and non-specific components of the cellular immune responses with a growing tumor. The model involves a combination of reaction-diffusion equations for the nutrient species and numerous rules of evolution for the cellular automaton description of the various cell-types that comprise the tissue-tumor environment. The effects of the innate and specific immune responses on the growth of the two-dimensional tumor are explored. Model components are represented by

- $N_{1}$, mitosis nutrient concentration

- $N_{2}$, survival nutrient concentration

- $H$, host cell number

- $T$, tumor cell number

- $I$, total immune cell number: the sum of the separate NK and cytotoxic T lymphocyte (CTL) cell populations. 


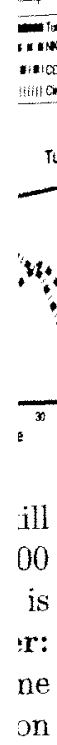

ill

00

is

s:

ne

on

or in both at extends s a spatial is has been is (PDEs),

ion of spafinite state sional spaJlism rules ire. In our riables are ent limited pecific (ina a growing for the nuJescription The effects imensional

1 cytotoxic
Parameter choices distinguish among the actions of the cells. The parameters involved in this model are given in detail in $[45]$.

The detailed CA rules are too extensive even to summarize here, but briefly: Each tumor cell is given the opportunity to move or divide according to the state of the cell's neighbors and some probability distribution, and is killed when the local survival nutrient levels are too low. The immune system is modeled by the inclusion of both natural killer cells and cytolytic $\mathrm{T}$ lymphocytes, both of which are able to lyse cancer cells. However, in keeping with the different functions of the innate and specific responses, the innate response of the NK cells is always present at low levels, and acts as a "first responder," while the T cells respond after a delay, first needing to be primed and recruited to the tumor site before becoming effective.

The diffusion of the chemical species is modeled through PDEs. As in the work of Ferreira et al. [22], [23], here we consider two nutrient species (such as glucose and oxygen $[26,67]$ ) - the first nutrient being a necessary component of the cell division processes, while the second is essential for cellular survival. The nutrients diffuse throughout the tissue space, and as they do so they are consumed by the different cells that are resident in tissue. The PDEs describing the dynamics of the chemical concentrations all have the following general mass-balance form:

(Time change in chemical concentration) $=$

(Chemical diffusion in space) - (Chemical consumption by each cell population).

Specifically, the nutrient species in this model are governed by the following partial differential equation:

$$
\underbrace{\frac{\partial N_{i}}{\partial t}}_{\text {nge in time }}=\underbrace{D_{i} \nabla^{2} N_{i}}_{\text {Diffusion }}-\underbrace{k_{1_{i}} H N_{i}-k_{2_{i}} T N_{i}-k_{3_{i}} I N_{i}}_{\text {Consumption }},
$$

where $N_{i}$ represents the concentration of proliferation $(i=1)$ or survival $(i=2)$ nutrient. The cell species are identified by $H$ for host cells (normal tissue), $T$ for tumor cells, and $I$ for immune cells. Also, $D_{i}$ is the diffusion coefficient for the nutrient, while the constants $k_{1_{i}}, k_{2_{i}}$ and $k_{3_{i}}$ are the rates of consumption of nutrient by host cells, tumor cells and immune cells, respectively.

In the absence of the immune system, the model is able to reproduce tumors of both compact-circular and papillary morphologies. These tumor shapes have direct dependence on the relative rates of consumption of the survival and mitosis nutrients by both tumor and host tissue cells. For circular tumors, the two-dimensional analog of the spherical tumor, simulations show the presence of necrotic cores and outer bands of proliferating cells. These results correspond qualitatively with the experimental literature (such as Folkman [26]).

The action of the immune system in this model leads to a variety of outcomes that depend on the choice of $T$ cell recruitment and death parameters. For virtually all immune system parameter choices, the resulting solutions displayed oscillatory behavior for tumor and immune cell populations in a similar manner to the purely temporal results of Kirschner and Panetta [37] where oscillations were also observed in effector and tumor cell populations. Experimental evidence for such oscillatory behaviour can also be found in works such as those due to Krikorian et al. [39] regarding non-Hodgkin's lymphoma.

Among other things, we used this model to demonstrate $\mathrm{T}$ cell infiltration into tumor growths. Experimental literature has shown that greater survival rates 


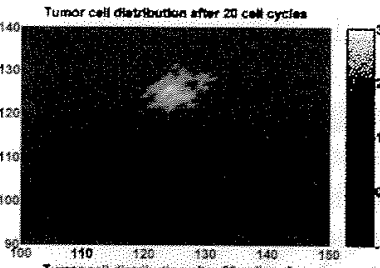

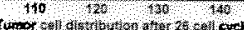
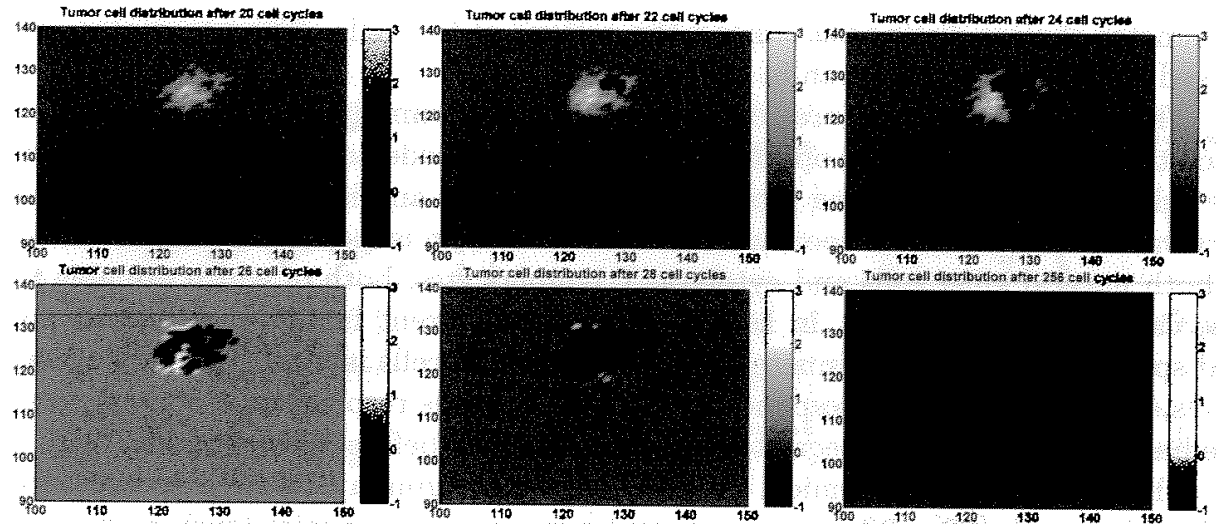

Figure 5. Six time frames of immune cell infiltration into a growing tumor leading to tumor destruction. Heavy damage is inflicted early in the tumor growth process. A grey scale of -1 indicates necrotic cells.

are observed in patients with lymphocyte infiltrates in tumors. For this model, we determined the parameter regimes in which infiltration is effective in tumor destruction. It is the subject of another of our studies to model the effects of injected $T$ cells and $T$ cell infiltration on pre-grown tumors.

Figure 5 shows the infiltration of $\mathrm{T}$ cells into the growing tumor over time and the resulting tumor destruction. In this simulation, a high $\mathrm{T}$ cell recruitment and low death rate cause an early and strong immune response to the young tumor, as seen in Figure 5 (second row, center panel). Over the remainder of the period of growth, up until total destruction at iteration 256 , the tumor cell population undergoes oscillatory growth. The tumor cells grow away from the immune cells in an attempt to evade them, while the immune cells are constantly directed to surround and kill the tumor cells. Eventually the cell division of the tumor cells is not sufficient to exceed the level of cell lysis by immune cells and the tumor is destroyed.

Further details of this $\mathrm{CA}$ model and a variety of numerical experiments are provided in $[\mathbf{4 5}]$.

\section{Micro-environment Driven Hybrid CA Model}

Current mathematical models do not adequately consider the effects of the cellular micro-environment on the metabolism of tumor cells. Evidence suggests that local conditions, such as nutrient availability, oxygen concentration, and local $\mathrm{pH}$, play a key role in tumor behavior, having significant effects on tumor growth, malignancy, and response to treatment $[\mathbf{7 3}],[\mathbf{6 3}]$. We have developed a preliminary model of tumor growth in tissue that describes the diffusion of chemical species through the tissue, the consumption of nutrients, the production of energy, and the production and elimination of metabolic waste. This preliminary model also describes tumor and normal cell proliferation and tumor necrosis and invasion in order to describe the system at the macroscopic level.
In this model pendent on local ulations of the mc oxygen concentrat parameters have $\mathrm{t}$ with observations The model descril the surrounding $t$ by-products. By c presence of the ti "health" that coul with the techniqu tumor growth can induced resistance the treatment on,

\subsection{Modelir} eral recent tumor centrations, ([16], ([3]). See, for exan ences therein, for o vascular stress, [5] interdependency $b$ The model current via micro-vessels, rounding cells, the of cellular energy of acidic byproduc

6.1.1. Oxygen, consume a variety ، and glucose are th sume, as in [58] th rely on anaerobic $I$ oxygen availability in 1929 by Warbur to recent experime notype (see, e.g., G consume a marked] become a very pop with varying degre tion supports the received attention low oxygenation an survival. We belie some of the remain

Oxygen is del but with increasin: a decrease in oxyg neously within the 
In this model, we assume that fuel consumption and ATP production are dependent on local oxygen concentration, available fuel concentration, and $\mathrm{pH}$. Simulations of the model are compared with the collected experimental data showing oxygen concentrations, $\mathrm{pH}$ and tissue morphologies, and calibration of the model parameters have been performed so that the output of simulations are consistent with observations from both in vitro and in vivo studies (e.g. [61], [35], [31]). The model describes the dependency of cell metabolism on the vascularization of the surrounding tissue as well as the concentrations of nutrients and metabolic by-products. By calculating available energy, the model predicts the effect of the presence of the tumor on the host, suggesting possible holistic quantifications of "health" that could be used as constraints in the optimization of therapies (in line with the techniques we used in [11] and [12]). The implemented model of cancer tumor growth can be used to suggest and test methods for dealing with hypoxiainduced resistance to treatments and the effect of the size of the molecules used in the treatment on delivery [24].

6.1. Modeling tumor metabolism, growth, and vascular collapse. Several recent tumor models allow cellular behavior to depend on local chemical concentrations, $([\mathbf{1 6}],[\mathbf{5 8}],[\mathbf{1 6}],[\mathbf{5 8}])$, and some models also include vascular dynamics, $([\mathbf{3}])$. See, for example, the excellent review by Araujo and McElwain, and the references therein, for other mathematical models of vascular tumors and tumor-induced vascular stress, $[\mathbf{5}]$. This model adds to these earlier models by incorporating the interdependency between tumor cell metabolism and local chemical concentrations. The model currently takes into consideration the delivery of oxygen and other fuels via micro-vessels, the constriction of blood vessels due to pressure from the surrounding cells, the synthesis of nutrients for the production of ATP, the budgeting of cellular energy towards growth and maintenance processes, and the production of acidic byproducts during glycolysis.

6.1.1. Oxygen, cell metabolism and tumor-induced hypoxia. While tumor cells consume a variety of fuels including glutamine, palmitate, oleate and others, oxygen and glucose are the most significant contributors to cellular energy [32]. We assume, as in [58] that tumor metabolism is characteristically glycolytic, where cells rely on anaerobic pathways for the majority of ATP production, regardless of local oxygen availability. While the concept of a glycolytic phenotype was first proposed in 1929 by Warburg, ([78]), some controversy still surrounds these hypotheses due to recent experimental evidence that challenges the perception of a glycolytic phenotype (see, e.g., Guppy et al. [32] ). On the other hand, because tumor cells often consume a markedly high rate of glucose, PET (positron emission tomography) has become a very popular technique for detecting and measuring a variety of tumors, with varying degrees of success [29], and for many the popularity of PET detection supports the concept of a glycolytic phenotype. Tumor oxygenation has also received attention recently after some researchers have found correlations between low oxygenation and metastatic progression [66], treatment resistance, and patient survival. We believe that an accurate mathematical model may help to answer some of the remaining questions regarding the role of glycolysis in tumor growth.

Oxygen is delivered to tissue via an efficient network of microvasculature, but with increasing size, tumors often compromise blood flow $[6]$, which causes a decrease in oxygen perfusion leading to hypoxic regions distributed heterogeneously within the tumor $[\mathbf{7 3}],[\mathbf{5 6}],[\mathbf{6 2}]$. In severely hypoxic regions, metabolism 
is more inefficient and the tumor cells are unable to maintain cell function, becoming necrotic. This model extends previous work by simulating the progression of hypoxia, its influence on local cell metabolism and ATP production, and the consequences for solid tumor growth.

6.2. Model Description. Like the model in [45], summarized in section 5, this model is categorized as a hybrid $C A$ model, whose continuous state variables include the concentrations of oxygen, nutrient, and hydrogen ions. The model also includes discrete state variables: proliferating tumor cells, necrotic tumor cells, normal cells, and blood vessels. The values of each of these seven state variables are stored in seven grids that evolve according to the local rules imposed by the automaton. In contrast to the previous model described in Section 5, these rules are purely deterministic, and are derived from the following assumptions:

(1) Growth and maintenance of cells depends on the rate of cellular energy (ATP) metabolized from nearby nutrients $[\mathbf{3 2}],[\mathbf{7 2}]$.

(2) Proliferation and death rates depend on the micro-environment, not on the sizes of the population, or on direct competition between cell populations. [38]. Note that these two assumptions lead to a different mechanism for intracellular competition than that described by other mathematical models. (See, for example, any of the previously cited tumor models.)

(3) Nutrient consumption rates depend on $\mathrm{pH}$ levels, glucose and oxygen concentrations, [7]. Consumption of nutrients and production of metabolic waste are all interdependent, $[\mathbf{3 3}]$.

(4) Tumor cells are able to produce ATP glycolytically more easily than normal cells $[29],[28],[64],[44],[36]$.

(5) Tumor cells thrive in a more acidic environment, and compete indirectly with normal cells by increasing the acidity of the micro-environment.

(6) Oxygen, glucose and lactate diffuse through tissue $[\mathbf{1 8}],[\mathbf{1 6}]$.

(7) Vascular constriction and collapse occurs inside the tumor due to an increase in cell density.

(8) Tumor cells excrete adhesive factors that can create cell-cell adhesive forces.

6.2.1. Illustrative Example: Modeling the Consumption of Glucose. Assumptions (3) and (6) lead to the same general form of mass-balance PDEs describing the concentration of oxygen, glucose and hydrogen as were used for the chemical concentrations for the model in section 5 . However, the consumption terms now depend on the concentration of the other molecules, as well as on the size of the cell populations. To illustrate the development of equations used in the model, we briefly discuss the equation modeling the consumption of glucose. The equations describing the consumption of oxygen and the production of hydrogen follow along the same lines, and are described in more detail in a forthcoming manuscript.

We use a modification of the empirical equation for oxygen and glucose consumption derived by Casciari et al. in $[8]$ in which cell metabolism and growth rates for EMT6/Ro tumor cells were determined to be functions of local environmental factors such as glucose concentrations, oxygen availability, and $\mathrm{pH}$. In Equation $6.1 G, O$ and $H$ represent the concentrations of glucose, oxygen and hydrogen ions 
Inction, beprogression on, and the

$n$ section 5 , te variables 3 model also tumor cells, tte variables osed by the , these rules ins:

lular energy

$t$, not on the populations, mechanism lathematical models.) oxygen conof metabolic

ily than nor-

te indirectly ronment.

lue to an incell adhesive se. AssumpIs describing the chemical in terms now le size of the he model, we he equations I follow along nuscript.

glucose con growth rates nvironmental In Equation rydrogen wors (resp.) (in mol $\cdot \mathrm{cell}^{-1} \mathrm{~s}^{-1}$ ), and $P$ represents a particular cell population.

$$
\frac{\partial G}{\partial t}=-D \nabla^{2} G-\left(a_{G}+\frac{c_{\nu} b_{G}}{O+\gamma_{\nu}}\right)\left(\frac{G}{G+k_{G}}\right)\left(\frac{1}{H^{n}}\right) P
$$

with $\gamma_{\nu}=\frac{c_{\nu} b_{G}}{q_{\nu} H^{n}-a_{G}}$. The dependence of consumption on cell-type is introduced through the two terms $c_{\nu}$ and $q_{\nu}$, where $\nu$ indicates the type of cell found at the location in question: proliferating tumor cell, necrotic tumor cell, or normal cell. This allows the model to reflect the assumption that normal cells should consume less glucose than tumor cells as oxygen levels decrease, as is seen experimentally with PET scans, $[\mathbf{5 4}]$. Oxygen consumption is calculated in a similar fashion, which then allows the computation of local ATP production. The production of $\mathrm{H}^{+}$ions is calculated as a by-product of the cell's metabolism, and is a function of the number of oxygen and glucose molecules consumed per unit of ATP produced. For the implementation of the model, the continuous PDEs describing the diffusion of oxygen, glucose and hydrogen ions (see equation 6.1) are translated into discrete time rules based on local concentrations. These small molecules diffuse on a time scale much faster than cell migration or proliferation. In the model of section 5 as well as in other works (see, e.g., [22], [23], [58]), it is assumed that the concentrations achieve a steady state distribution between each cell proliferation step, so a time-independent system of PDEs is solved at each step. In contrast, in this model, the computational step implementing diffusion still incorporates time dependence, allowing the model to reflect the biological processes more accurately.

6.3. Model Simulations. Results from a simulation of the model are shown in Figure 6, in which two small tumors were initially placed in vascularized tissue. After 200 days the tumors have grown considerably, but not uniformly, and have developed necrotic cores. By calculating the total change in ATP production, the model can quantify changes in the energy available to the host. The graph of glucose levels (Figure 6, second row, left panel) highlights the prediction of dynamic energy budget (DEB) theory $[\mathbf{7 2}]$ that tumor cells will consume more glucose than normal cells. This reflects the assumption that tumor cells are able to metabolize glycolytically more readily than normal cells. Glycolysis produces more lactate and is less efficient than aerobic metabolism, and so the $\mathrm{O}_{2}$ concentrations and the pH of the tissue surrounding the tumor is decreased, and the tissue occupied by the tumor cells becomes hypoxic and more acidic (shown in Figure 6, bottom row, center and right panels). These changes in the micro-environment effected by the presence of the tumor have consequences not only for the normal cells and the overall energy budget of the host, but can also affect the delivery and bio-distribution of potential treatments, a factor that we propose to explore with this model.

6.4. Extensions of the hybrid-CA model. The two hybrid-CA models described in Sections 5 and 6 will be combined, so that effects of the microenvironment on the effectiveness of the immune response and therapy can be explored. In particular, treatment of solid tumors with nano-vaccines could be simulated, suggesting protocols that could be tested in the laboratory.

In the avascular phase, solid tumor growth is limited by the ability of nutrients to diffuse through more than a few cell diameters. In order to continue to grow, a solid tumor must induce new vascular growth through angiogenesis, a process crucial to tumor malignancy. With this in mind, we will extend our model to include 

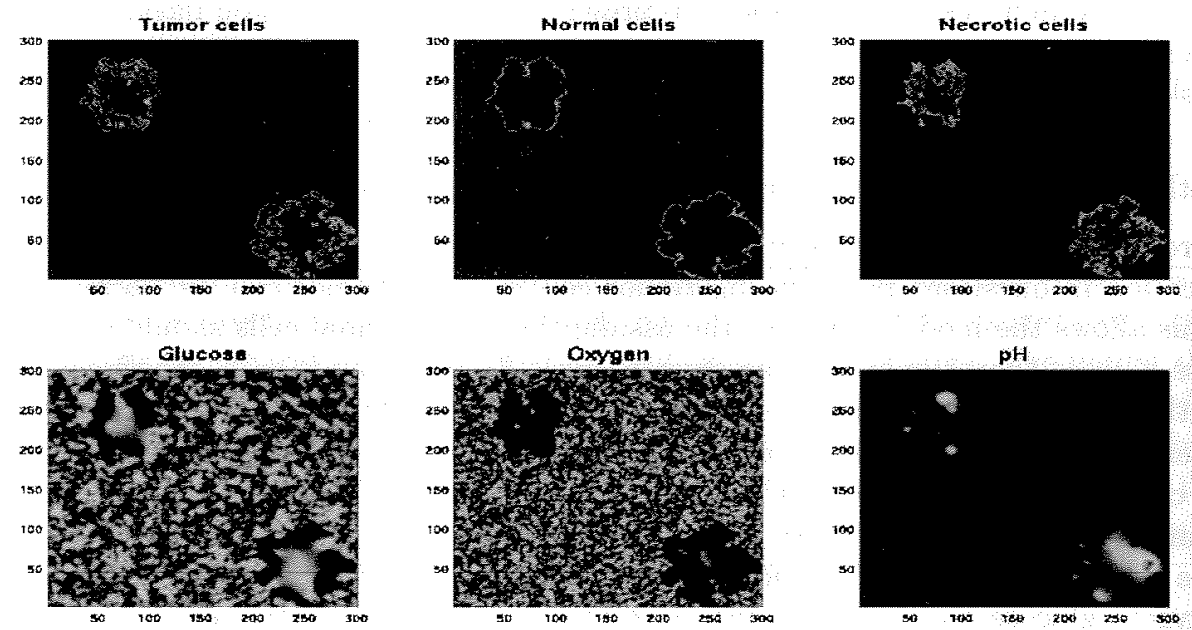

FIGURE 6. Simulation showing two tumors with necrotic cores. The panels from left to right, top to bottom, show concentrations of tumor cells, normal cells, necrotic cells, glucose, oxygen and $\mathrm{pH}$.

angiogenesis. This requires modeling the production and diffusion of angiogenic growth factor (AGF), and the chemotactic movement of capillaries. We plan to build on models described in, for example [4], [47], and [77]. We will add the energy requirements for the construction of new vasculature to the total energy budget, and in this way tie the local energy requirements to global demands on the host.

\section{Conclusion}

In the quest to develop models of tumor growth that hold the promise of becoming clinically relevant, we have presented a progression of models, both developed and in development, each with a focus that provides insights into how to harness the potential of combination therapies working with the immune system in controlling a growing tumor. Optimal control theory, as an aid to searching for optimized treatment strategies, has been been employed in the earlier models, while our more recently developed models have been designed to allow for the application of an optimal control strategy that can employ state constraints as a means of minimizing the damaging side effects of chemotherapy $[\mathbf{1 1}],[\mathbf{1 2}],[13],[14],[10]$. Our models that employ a spatial component will also admit the addition of a vascular network that can allow for spatially dependent drug, nutrition and immune cell delivery, as well as consideration of antiangiogenic therapy [45]. Adhesivity factors are included so that metastasis of the primary tumor can be modeled. Furthermore, the effect of the disease on the energy-level of the host can be examined through the inclusion of a glycolytic phenotype and the calculation of the available ATP. As always in the modeling process, the proper balance of complexity and analytical tractability must be maintained, with the exact mix depending on the biological question that drives the model itself.
1. P.A. Abrams and or neither?, TREE

2. H.R. Akcakaya, R that works, Ecolog

3. T. Alarcon, Byrne inhomogeneous ene

4. A. R. A. Anderson tumor-induced ang

5. R.P. Araujo and D. Mathematical Biolc

6. ___ New insigh Theoretical Biology

7. J.J. Casciari and J. cellutar spheroids fi no. $1,28-36$.

8. J.J. Casciari, S.V. \& metabolism with ox of Cellular Physiolo

9. C. Colijn and M. M ogenous leukemia,

10. L.G. de Pillis, W. tumors: Modeling a (2005), in press.

11. L.G. de Pillis and and drug therapy:

12. __ The dynam Model. (Special Issu

13. ___ Immune re Bathe, ed.), vol. 2,

14. L.G. de Pillis, A.E. mediated immune $r$

15. A. Diefenbach, E.R NKG2D receptor sti

16. S. Dormann and A. cellular automaton.

17. M.E. Dudley, J.R. W Topalian, R. Sherry, Seipp, L. Rogers-Fre Cancer regression a lymphocytes, Science

18. A. Dutta and A.S. I Theoretical Biology

19. A. Ergun, K. Camph inhibitors, Bulletin

20. Miklos̀ Farkas, Dyno

21. J.D. Farrar, K.H. Ka Cancer dormancy. and maintaining the

22. S. C. Ferreira, M. I avascular tumor, $\mathrm{Ph}$

23. Morphology 67 (2003), 051914.

24. T. Fifis, A. Gamvrel and M. Plebanski, $S$ nano-vaccines again 


\section{References}

1. P.A. Abrams and L.R Ginzburg, The nature of predation: prey dependent, ratio dependent or neither?, TREE 15 (2000), no. 8, 337-341.

2. H.R. Akcakaya, R. Arditi, and L.R. Ginzberg, Ratio-dependent predation: An abstraction that works, Ecology 76 (1995), no. 3, 995-1004.

3. T. Alarcon, Byrne H.M., and P.K. Maini, A cellular automaton model for tumour growth in inhomogeneous environment., Journal of Theoretical Biology 225 (2003), 257-274

4. A. R. A. Anderson and M. A. J. Chaplain, Continuous and discrete mathematical models of tumor-induced angiogenesis, Bulletin of Mathematical Biology 60 (1998), 857900.

5. R.P. Araujo and D.L.S. McElwain, A history of the study of solid tumour growth, Bulletin of Mathematical Biology 66 (2004), no. 5, 1039-1091.

6. N New insights into vascular collapse and growth dynamics in solid tumors, Journal of Theoretical Biology 228 (2004), no. 3, 335-346.

7. J.J. Casciari and J.S. Rasey, Determination of the radiobiologically hypoxic fraction in multicellular spheroids from data on the uptake of 3Hfluoromisonidazole, Radiat Res. 141 (1995), no. $1,28-36$.

8. J.J. Casciari, S.V. Sotirchos, and R.M. Sutherland, Variations in tumor cell growth rates and metabolism with oxygen concentration, glucose concentration, and extracellular ph., Journal of Cellular Physiology 151 (1992), 386-394.

9. C. Colijn and M. Mackey, A mathematical model of hematopoiesis - i. periodic chronic myelogenous leukemia, Journal of Theoretical Biology in press (2005), 1-16.

es.

ins

H.

angiogenic We plan to ill add the otal energy ands on the

ie of becom1 developed to harness 1 in controlr optimized le our more on of an opminimizing Our models lar network delivery, as are included the effect of he inclusion is always 11 tractabilitw uestion that

10. L.G. de Pillis, W. Gu, and A.E. Radunskaya, Mixed immunotherapy and chemotherapy of turnors: Modeling applications and biological interpretations, Journal of Theoretical Biology (2005), in press.

11. L.G. de Pillis and A.E. Radunskaya, A mathematical tumor model with immune resistance and drug therapy: an optimal control approach, J Theor Med. 3 (2001), $79-100$.

12. The dynamics of an optimally controlled tumor model: A case study, Math Comput Model. (Special Issue) 37 (2003), no. 11, 1221-1244.

13. ___ Immune response to tumor invasion, Computational Fluid and Solid Mechanics (K.J. Bathe, ed.), vol. 2, M.I.T., 2003, pp. 1661-1668.

14. L.G. de Pillis, A.E. Radunskaya, and C.L. Wiseman, A validated mathematical model of cellmediated immune response to tumor growth, Cancer Research 65 (2005), 70507958.

15. A. Diefenbach, E.R. Jensen, A.M. Jamieson, and D. Raulet, Rael and H60 ligands of the NKG2D receptor stimulate tumor immunity, Nature 413 (2001), 165-171.

16. S. Dormann and A. Deutsch, Modeling of self-organized avascular tumor growth with a hybrid cellular automaton., In Silico Biology 2 (2002), 0035.

17. M.E. Dudley, J.R. Wunderlich, P.F. Robbins, J.C. Yang, P. Hwu, D.J. Schwartzentruber, S.L. Topalian, R. Sherry, N.P. Restifo, A.M. Hubicki, M.R. Robinson, M. Raffeld, P. Duray, C.A. Seipp, L. Rogers-Freezer, K.E. Morton, S.A. Mavroukakis, D.E. White, and S.A. Rosenberg, Cancer regression and autoimmunity in patients after clonal repopulation with antitumor lymphocytes, Science 298 (2002), no. 5594, 850-854.

18. A. Dutta and A.S. Popel, A theoretical anlaysis of intracellular axygen diffusion, Journal of Theoretical Biology 176 (1995), 433-445.

19. A. Ergun, K. Camphausen, and L.M. Wein, Optimal scheduling of radiotherapy and angiogenic inhibitors, Bulletin of Mathematical Biology 65 (2003), no. 3, 407-424.

20. Miklos Farkas, Dynamical models in biology, Academic Press, San Diego, CA, 2001.

21. J.D. Farrar, K.H. Katz, J. Windsor, G. Thrush, R.H. Scheuermann, J.W. Uhr, and N.E. Street, Cancer dormancy. VII. A regulatory role for CDS $T$ cells and IFN-gamma in establishing and maintaining the tumor-dormant state, J Immunol. 162 (1999), no. 5, 2842-9.

22. S. C. Ferreira, M. L. Martins, and M. J. Vilela, Reaction-diffusion model for the growth of avascular tumor, Phys Rev E 65 (2002), 021907

23. _....... Morphology transitions induced by chemotherapy in carcinomas in situ, Phys Rev E 67 (2003), 051914 .

24. T. Fifis, A. Gamvrellis, B. Crimmen-Irwin, G. Pietersz, J Li, P. Mottram, I.F.C. Mckenzie and M. Plebanski, Size-dependent immunogenicity: Therapeutic and protective properties of nano-vacines against tamors, Journal of Immunology (2004), 3148-3154. 
25. K.R. Fister and J.H. Donnelly, Immunotherapy: An optimal control approach, Mathematical Biosciences and Engineering 2 (2005), no. 3, 499-510.

26. J. Folkman and M. Hochberg, Self-regulation of growth in three dimensions, Journal of Ex. perimental Medicine 138 (1973), 745-753.

27. S. N. Gardner, A mechanistic, predictive model of dose-response curves for cell cycle phase specific and nonspecific drugs, Cancer Res. 60 (2000), 1417-1425.

28. R.A. Gatenby and E. T. Gawlinski, The glycolytic phenotype in carcinogenesis and tumbr invasion. Insights through mathematical models, Cancer Res. 63 (2003), 3847-3854.

29. R.A. Gatenby and E.T. Gawlinski, The glycolytic phenotype in carcinogenesis and tumor invasion: Insights through mathematical models., Cancer Research 63 (2003), 3847-3854.

30. R. Glas, L. Franksson, C. Une, M. Eloranta, C. Ohlen, A. Orn, and K. Karre, Recruitment and activation of natural killer (NK) cells in vivo determined by the target cell phenotype: An adaptive component of NK cell-mediated responses, J. Exp. Med. 191 (2000), no. 1, 129138.

31. M. Gryczynskia, J. Kobosb, and Pietruszewska W., Intratumoral microvessels density and morphometric study of angiogenesis as prognostic factor in laryngeal cancer, International Congress Series $1240(2003), 1113-1118$.

32. M. Guppy, P. Leedman, X. Zu, and V. Russel, Contribution by different fuels and metabolic pathways to the total atp turnover of proliferating mcf-7 breast cancer cells., Biochem. J. 364 (2002), 309-315.

33. G. Helmlinger, A. Sckell, M. Dellian, N.S. Forbes, and R.K. Jain, Acid production in glycolysis. impaired tumors provides new insights into tumor metabolism, Clinical Cancer Research 8 $(2002), 1284-1291$

34. J.F. Holland and F. Emil III (eds.), Cancer medicine, ch. II-3, XII, XV, Lea and Febiger. 1973.

35. M.E. Hystad and E.. Rofstad, Oxygen consumption rate and mitochondrial density in human melanoma monolayer cultures and multicellular spheroids., Int J Cancer. $\mathbf{5 7}$ (1994), no. 1. $532-7$.

36. J.P. Kirkpatrick, D.M. Brizel, and M.W. Dewhirst, A mathematical model of tumor oxygen and glucose mass transport and metabolism with complex reaction kinetics, Radiation Research 159 (2003), 336-344

37. D. Kirschner and J.C. Panetta, Modeling immunotherapy of the tumor-immune interaction. J Math Biol. 37 (1998), no. 3, 235-52.

38. S.A.L.M. Kooijman and L.A. Segel, How growth affects the fate of cellular metabolited, Bul letin of Mathematical Biology 67 (2005), 57-77.

39. J.G. Krikorian, C.S. Portlock, D.P. Cooney, and S.A. Rosenberg, Spontaneous regression of non-hodgkin's lymphoma: A report of nine cases, Cancer 46 (1980), 2093-2099.

40. V. Kuznetsov, I. Makalkin, M. Taylor, and A. Perelson, Nonlinear dynamics of immunogenit tumors: Parameter estimation and global bifurcation analysis, Bulletin of Mathematical Biology 56 (1994), no. 2, 295-321.

41. V.A. Kuznetsov and G.D. Knott, Modeling tumor regrowth and immunotherapy, Math Comput Model. 33 (2001), 1275-1287

42. U. Ledzewicz and H. Schattler, Drug resistance in cancer chemotherapy as an optimal control problem, Discrete and Continuous Dynamical Systems - Series B 6 (2006), no. 1, 129150.

43. Y Liang, K.S. Leung, and T.S.K. Mok, Evolutionary drug scheduling model for cancer chemotherapy, Genetic and Evolutionary Computation (GECCO) 3103 (2004), 1126-1137.

44. H Lu, RA Forbes, and A. Verma, Hypoxia-inducible factor 1 activation by aerobic glycolysts implicates the Warburg effect in carcinogenesis., J Biol Chem. 277 (2002), no. 26, 23111-3

45. D. Mallet and L.G. de Pillis, A cellular automata model of tumor-immune system interactions. Journal of Theoretical Biology (2005), in press.

46. J. McCall, Genetic algorithms for modelling and optimisation, Journal of Computational and Applied Mathematics 184 (2005), no. 1, 205-222.

47. S. McDougall, A. Anderson, M. Chaplain, and J. Sherratt, Mathematical modelling of for through vascular networks: implications for tumor-induced angiogenesis and chemotherapy strategies, Bulletin of Mathematical Biology 64 (2002), 673-702

48. B. Melichar, J. Dvorak, P. Jandik, M. Touskova, D. Solichova, J. Megancova, and Vobonl 7. Intraarterial chemotherapy of malignant melanoma metastatic to the liver, Hepatogastroenterology 48 (2001), no. 42, 1711-1715. 
49. S. Michelson and J.T. Leith, Host response in tumor growth and progression, Invasion and Metastasis 16 (1996), no. 4-5, 235-246.

tal of Ex-

50. S. Morecki, T. Pugatsch, S. Levi, Y. Moshel, and S. Slavin, Tumor-cell vaccination induces tumor domancy in a murine model of B-cell leukemia lin , Tumor-cell vaccination induces (1996), no. 2, 204-8.

51. M. Muller, F. Gounari, S. Prifti, H.J. Hacker, V. Schirmacher, and K. Khazaie, Eblacz tumor dormancy in bone marrow and lymph nodes: active control of proliferating tumar cells by CD8+ immune T cells, Cancer Res. 58 (1998), no. 23, 5439-46.

52. J. M. Murray, Mathematical biology, second ed., Springer-Verlag, Berlin, 1993

53. M.M. Mustafa, G.R. Buchanan, N.J. Winick, G.H. McCracken, I. Tkaczewski Q. Ansari, and M.S. Agopian, Immune reco, G.H. McCracken, I. Tkaczewski, M. Lipscomb, of chemotherapy. I Pediatr of chemotherapy, J Pediatr Hematol Oncol. 20 (1998), no. 5, 451-457.

54. Y. Nakamoto, K.R. Zasadny, H. Heikki Minn (1998), no. 5, 451-457. of common semi-quantitative parameters for and R.L. Richard L. Wahl, Repraducibility positron emission tomography using for evaluating lung cancer glucose metabolism with Biology 4 (2002) tomography using 2-deoxy-2-/18 f/fluoro-d-glucose, Molecular Imaging and

tionship between angiogehlesh, M. J. Browning, W. P. Steward, and A. L. Harris, The relaof malignant disease, Egenesis and the immune response in carcinogenesis and the progression of malignant disease, Eur J Cancer. 36 (2000), 151-169.

56. M. Owen, H. Byrne, and C. Lewis, Mathematical modelling of the use of macrophages as vehicles for drug delivery to hypoxic tumour sites, Journal of Theoretical Biology 226 (2004) 377-391.

57. M.R. Owen and J.A. Sherratt, Modelling the macrophage invasion of tumours: Effects on growth and composition, IMA Journal of Mathematics Applied in Medicine and Biology 15
(1998), 165 185.

58. A.A. Patel, E.T. Gawlinski, S.K. Lemieux, and R.A. Gatenby, A cellular automaton model of early tumor growth and invasion: The effects of native tissue vascularity and increased anaerobic tumor metabolism, Journal of Theoretical Biology 213 (2001), 315-331.

59. R. Pazdur, W. Hoskins, L. Wagman, and L. Coia (eds.), Cancer management: A mulitdisciplinary approach, eighth ed, ch. Principles of Chemother apy, Oncology Publishing Group of CMP Healthcare Media, 2004, Available at http://www.cancernetwork.com/handbook/contents.htm Accessed May 2005.

60. A.E. Radunskaya and M. Villasana A delay differentiol Accessed May 2005. J.Math.Biol. 47 (2003), 270-294. poxia in human melanoma xenografts: agical and immunohistochemical assessment of hyJ Radiat Biol. 75 (1999), no. 11,1377 acute and chronic hypoxia in individual tumours., Int

62 Sansone BC Scalerand), Mo. 11, 1377.93.

Letters 89 (2002)

21, Art. No. 218101

$p H$ of tumors., Neoplasia 5 (2003) nutions of cell metabolism and $H^{+}$diffusion to the acidic Journal of Mathematical

65. T.H. Stewart Imment no. $1,74-82$

66. P Subarsky and RP Hill, The hypoxic tumour microenvironment and metastatic progression Clinical \& Experimental Metastasis 20 (2003), no. 3, 237-250.

67. R.M. Sutherland, Cell and environment interactions in tumor microregions. the multicell spheroid model, Science 240 (1988), 177-184.

68. A. Swierniak, Some control problems for simplest differential models of proliferation cycle. Applied Mathematics and Computer Science 4 (1994), no. 2, 223-232.

69. A. Swierniak and A. Polanski, Irregularity in scheduling of cancer chemothernot Mathematics and Computer Science 4 (1994), no. 2, 263-271.

70. A. Swierniak, A. Polanski, and M. Kimmel, Optimal control problems arising in cell-cycle specific cancer chemotherapy, Journal

71. A Swierniak and J. Smeija, Journal of Cell Proliferation 29 (1996), 117-139. A. Swierniak and J. Smeija, Analysis and optimization of drug resistant and phase-specifbe cancer, Mathematical Biosciences and Engineering 2(2005), no. 3,657 670 
72. IMM. van Leeuwen, C. Zonneveld, and SALM. Kooijman, The embedded tumour: host physiology is important for the evalabtion of tumour growth., British Journal of Cancer $\mathbf{8 9}(2003)$. $2254-2263$.

73. P Vaupel, O Thews, DK Kelleher, and M. Hoeckel, Current status of knowledge and critical issues in tumor oxygenation. Results from 25 years research in tumor pathophysiology, Adv Exp Med Biol. 454 (1998), 591-602.

74. M. Villasana and G. Ochoa, Heuristic design of cancer chemotherapies, IEEE Transactions on Evolutionary Computation 8 (2004), no. 6, 513-521.

75. Oskar von Stryk, User's guide for DIRCOL: A direct collocation method for the numerical solution of optimal control problems, Lehrstuhl M2 Numerische Mathematik, Technische Universitaet Muenchen, September 1999, Copyright (C) 1994-1999 Technische Universitaet: Muenchen.

76. C.J. Wheeler, D. Asha, L. Gentao, J.S. Yu, and K.L. Black, Clinical responsiveness of glioblastoma multiforme to chemotherapy after vaccination, Clin Cancer Res. 10 (2004), 5316-5326.

77. X. Zheng, S.M. Wise, and V. Cristini, Nonlinear simulation of tumor necrosis, neovascularization and tissue invasion vio an adaptive finite-element/level-set method. Bulletin of Mathematical Biology 00 (2003), 1-53.

78. X.L. Zu and M. Guppy, Cancer metabolism: facts, fantasy, and fiction., Biochemical and Biophysical Research Communications. 313 (2004), 459-465

Departant of Mathematics, Harvey mudo College, Claremont, CA 91711

E-mail address: depillisohme ,edu

Department of Mathematics, Pomona College, Claremont, CA 91711

E-mail address: aradunskayagpomona.edu

Impact

\begin{abstract} carcinoma and warts are expect determinis vaccine th "infectious infective- $r$ thresholds sure of vac number is and a unic less than methods to well as con
\end{abstract}

Genital infect fection among sex current infections Although the ma certain HPV typ lesions. HPV infe condylomata, anc health burden anc [29]).

2000 Mathemati Key words and stability, endemic equ

The author wish Modeling the Dynam for the opportunity tc (Merck Research Lab This paper is in 